\title{
POEMS Syndrome Manifested as Systemic Capillary Leak Syndrome: A Case Report
}

\author{
Ana Carolina Peçanha Antonio ${ }^{\mathrm{a}, \mathrm{b}}$, Gustavo Adolpho Moreira Faulhaber ${ }^{\mathrm{a}}$, \\ Tania Weber Furlanetto ${ }^{\mathrm{a}}$
}

\begin{abstract}
The authors report a case of an old woman who presented with a history of repetitive dyspnea and inferior limbs edema episodes, up to the point she showed extreme anasarca, hypotension, hypoalbuminemia, hemoconcentration and cachexia, developing unexplained respiratory insufficiency until death, in spite of all interventions. A diagnosis was obtained by autopsia: multicentric Castleman's disease manifested as capillary leak syndrome and possible POEMS syndrome (polyneuropathy, organomegaly, endocrinopathy, M protein and skin changes), all conditions of rare incidence and of an intriguing association.
\end{abstract}

Keywords: Capillary-leak syndrome; POEMS syndrome; Castleman disease; Monoclonal gammopathy

\section{Introduction}

POEMS syndrome is a rare paraneoplastic condition secondary to a plasma cell dyscrasia, mainly Castleman Disease. Recognition of a complex combination of its manifestations in a scenario of generalized capillary leak is a huge challenge and at least partially accounts for its poor prognosis.

\section{Case Report}

A 71-year-old white female was admitted to the hospital for evaluation of generalized subcutaneous edema and abdomi-

\footnotetext{
Manuscript accepted for publication March 21, 2013

${ }^{a}$ Internal Medicine Division, Hospital de Clinicas de Porto Alegre, Universidade Federal do Rio Grande do Sul, Porto Alegre, RS, Brazil

${ }^{\mathrm{b}}$ Corresponding author: Ana Carolina Pecanha Antonio, Ari Marinho, 11/210, Zip Code: 90520-300, Porto Alegre, RS, Brazil.

Email: ana.carolina.pecanha@me.com

doi: http://dx.doi.org/10.4021/jmc1217w
}

nal swelling that she noticed two weeks ago. She also complained of liquid diarrhea that improved partially avoiding milk ingestion, anorexia and a massive weight loss in the last six months. She had no fever or night sweats. In the past twelve months, she decreased progressively her daily activities, becoming increasingly dependent on others for her usual care, and remaining in bed almost 24 hours a day. She was brought two times to the emergency room in the last 30 days for dyspnea, orthopnea and symmetric leg edema, and was discharged with diagnosis of heart failure. She considered that her health had been good until 18 months ago, when she was admitted for an ischemic stroke that decreased slightly the strength in her right arm. She had a long history of arterial hypertension, and she has been treated for depression in the last two years. She never smoked or drank alcoholic beverages. Her current medications were aspirin, simvastatin, and sertraline. Her antihypertensive medications were withdrawn in her last appointment with her physician in the last week, because her blood pressure was low.

The physical exam revealed cachexia, normal mental status, no fever, blood pressure: $82 / 56 \mathrm{mmHg}$, heart rate: 92 $\mathrm{bpm}$, respiratory rate: $26 \mathrm{mpm}$. There was no jugular venous distension, nor hepatojugular reflux. The oxygen saturation value obtained from pulse oximetry was $95 \%$. There were inspiratory rales in the lower lung fields, bilateral and symmetric lower extremity edema, and ascites. Enlarged cervical lymph nodes with $1.5 \mathrm{~cm}$ were detected. Blood hemoglobin was $12.9 \mathrm{~g} / \mathrm{dL}$. Blood leukocytes and platelets counts, serum urea nitrogen, creatinine, AST, ALT, bilirubin, alkaline phosphatase, electrolytes, and TSH levels were normal. Serum albumin level was $1.9 \mathrm{~g} / \mathrm{dL}$. Serum immunoelectrophoresis revealed a $0.55 \mathrm{~g} / \mathrm{dL}$ monoclonal $\mathrm{IgG}$ lambda component. The erythrocyte sedimentation rate was $14 \mathrm{~mm} /$ hour (0 - 29 $\mathrm{mm} /$ hour). The urinalysis was normal. Serologic tests for HIV, HCV and HBV were negative. The first chest radiologic examination revealed bilateral reduced lung volume, and pleural effusion, with no signs of increased pulmonary or caval venous pressure, and normal cardiac area. Transthoracic two-dimensional echocardiography with Doppler showed an ejection fraction of $78 \%$, normal sized heart chambers, hypertrophy of the left ventricle, and decreased diastolic filling rate. The pulmonary arterial pressure was estimated between 


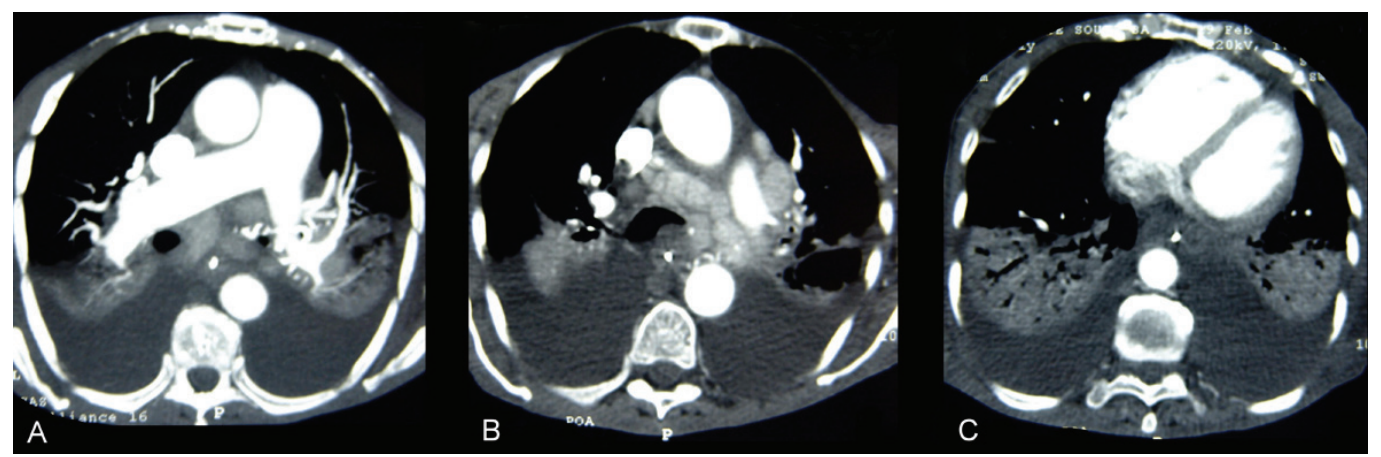

Figure 1. CT pulmonary angiogram had no evidence of pulmonary embolism (A), but disclosed multiple large mediastinal adenomegalies (B) and pleural effusion with atelectasis or consolidation in the adjacent area of the lungs (C).

45 and $50 \mathrm{mmHg}$. An abdominal ecography study disclosed ascites and splenomegaly $(13 \mathrm{~cm})$. Pleural and ascites fluid analysis were compatible with transudate in both locations. A chest computed angiotomography study showed large mediastinal lymph nodes $(1.5-2 \mathrm{~cm})$, bilateral pleural effusion, ground glass opacification in both lower lung fields and no evidence of pulmonary embolism, as shown in Figure 1. In a few days, the patient developed intense and progressive respiratory dysfunction. A chest RX disclosed diffuse filling of the alveolar spaces (Fig. 2), and was similar to the first exam in other aspects.

She was treated with oxygen, by nasal catheter and CPAP mask, intravenous fluids and glucocorticoids. No infection was identified by blood and urine cultures. Liquid was removed from pleural and peritoneal spaces with no relief. Despite medical care, the patient had a cardiac arrest, and did not respond to resuscitative efforts.

\section{Discussion}

Postmortem examination was compatible with multicentric Castleman's disease, plasma cell variant, involving lymph nodes, spleen and bone marrow (Fig. 3, 4). There was mod- erate myocardial hypertrophy and normal lungs, and large effusions to pleural, peritoneal and pericardial spaces. Based on the clinical presentation, capillary leak syndrome and possible POEMS syndrome were diagnosed.

POEMS Syndrome (polyneuropathy, organomegaly, endocrinopathy, $M$ protein and skin changes) is a rare syndrome whose major diagnostic criteria are polyneuropathy and monoclonal proliferative plasma cell disorder. The last condition must be present, either as osteosclerotic myeloma or Castleman's Disease. Such criteria were established by Dispenzieri et al in 2003, after evaluating 226 patients [1]. All had also polyneuropathy, manifesting initially as sensitive symptoms, and lately motor symptoms, with inability to walk or even seat [2]. The polyneuropathy was predominantly an axonal degeneration, similar to the chronic inflammatory demyelinating polyradiculoneuropathy [3]. In the series described by Dispenzieri, organomegaly (liver, spleen, lymph nodes), endocrinopathy (except diabetes and thyroidopathy, excluded for the large prevalence in the population), edema, skin alteration, and papilledema were found in $50 \%, 67 \%, 29 \%, 68 \%, 29 \%$, respectively [1]. Diarrhea, anorexia and marked weight loss were also described [4]. Pulmonary arterial hypertension occurred in $25 \%$ in one study [5], with systolic pressure varying from 50 to 65

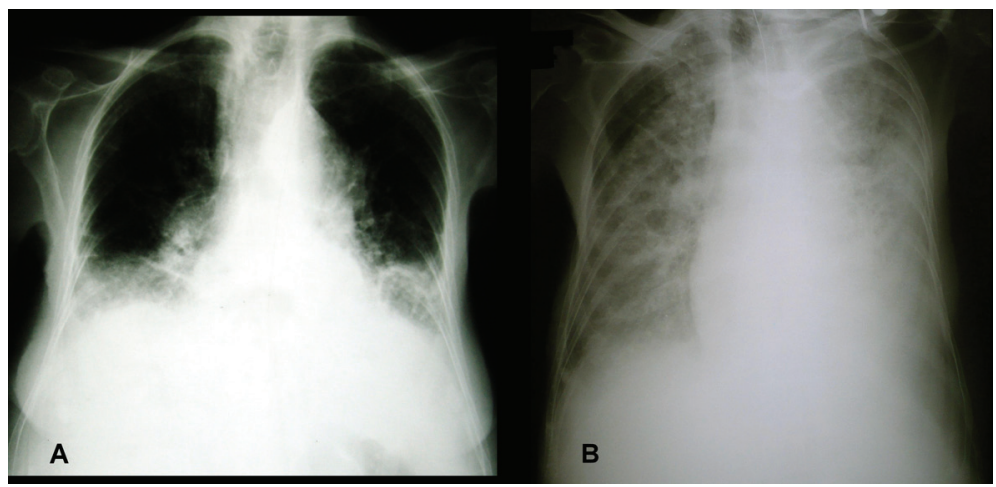

Figure 2. Chest RX showing diffuse alveolar filling. 


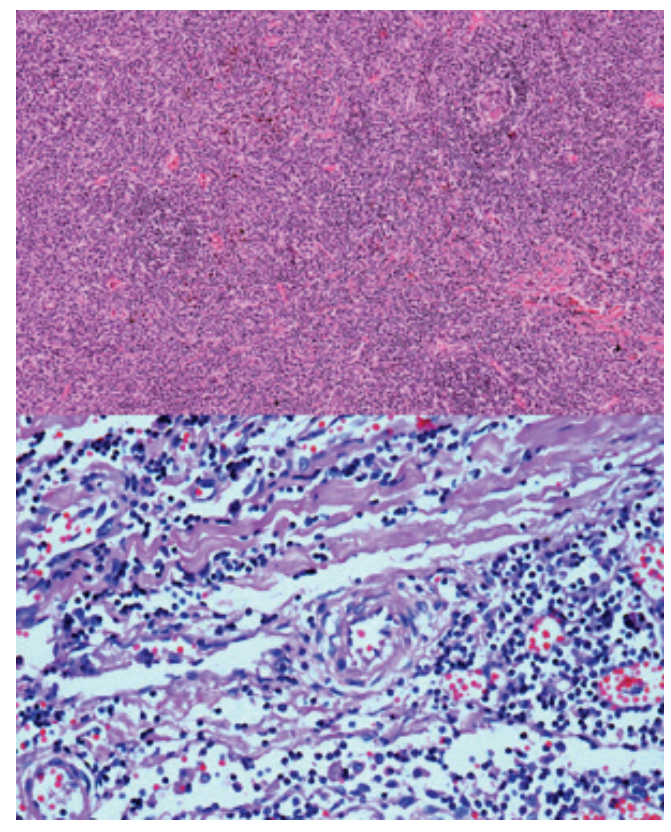

Figure 3. (Upper) Photomicrograph of mediastinal lymph node stained with HE (original magnification $100 \times$ ): note the reduced germinal center formation and loss of lymph node architecture by mononuclear cell proliferation into interfollicular spaces, as observed in the plasma cell variant of Castleman's Disease. (Lower) Photomicrograph of mediastinal lymph node showing deposition of hyaline amorphous material and concentric rings of small lymphocytes around vessels, with onion-skin appearance, $\mathrm{HE}, 400 \times$.

mmHg. Peripheral edema, ascites or pleural effusion were observed in $29 \%$ of patients, and predicted a bad prognosis in POEMS Syndrome [1]. The presence of at least two major and one minor criteria are required for diagnosis. In our patient, the progressive loss of the ability to walk and stand, with no evidence of a new stroke, with normal mentation, is highly suggestive of polyneuropathy.

Castleman's disease, also known as angiofollicular lymph node hyperplasia, is a lymphoproliferative disorder, characterized in its multicentric presentation by generalized lymphadenopathy, hepatoesplenomegaly, and constitutional symptoms, as fever, night sweats and weight loss. It can be part of the POEMS Syndrome. Multicentric Castleman's disease (MCD) is seen more commonly in patients infected with HIV, and infection with human herpesvirus-8 (HHV-8) has been described, both in patients infected with HIV or not $[6,7]$. Yamasaki et al [7] reported sixteen HIV-negative patients with MCD, and no Kaposi's sarcoma; in this series, the median age was 48 years and fifteen had systemic symptoms such as fever and fatigue. In $81 \%$ of these patients HHV-8 DNA was detected in lymph nodes, and all patients had the plasma cell variant of MCD.

The systemic capillary leak syndrome (SCLS) is associated with hypovolemic shock due to plasma leak, causing also hemoconcentration, hypoalbuminemia and anasarca,

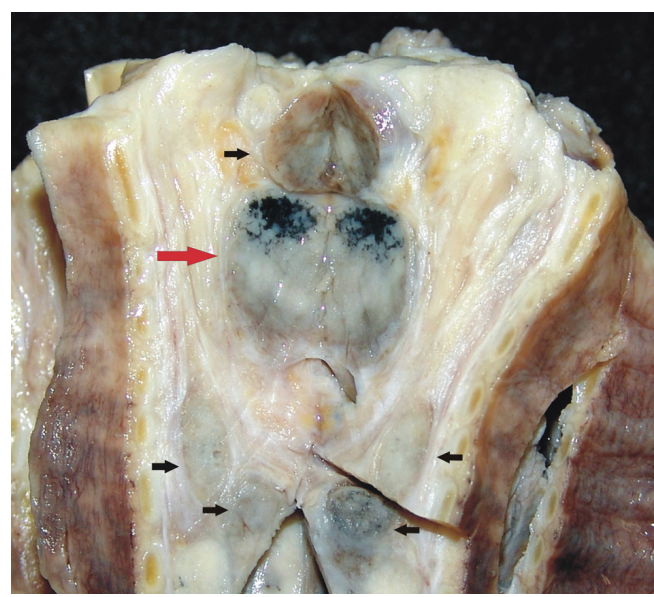

Figure 4. Longitudinal section of trachea showing large lymph nodes on the wall (arrows), in addition to anthracosis (red arrow).

due to a sudden shift of fluid and macromolecules from the intravascular to the interstitial space. All patients, with a few exceptions, have an IgG monoclonal gammopathy in serum. Pulmonary edema occurs in the recovery phase [8]. In MCD, increased serum vascular endothelial growth factor (VEGF) and interleukin 6 levels were associated with capillary damage and leak of plasma to interstitium, decreasing circulatory volume [9]. In 12 patients with MCD and HIV, two had adult respiratory distress syndrome [10].

The massive pulmonary edema with no evidences whatsoever of heart failure and the anasarca, observed in our patient, is compatible with the diagnosis of SCLS. The arterial hypotension observed could also be explained by this syndrome. Adrenal failure was not evaluated, nevertheless she did not respond to the administration of hydrocortisone.

\section{Funding}

None.

\section{Conflict of Interest}

None.

\section{References}

1. Dispenzieri A, Kyle RA, Lacy MQ, Rajkumar SV, Therneau TM, Larson DR, Greipp PR, et al. POEMS syndrome: definitions and long-term outcome. Blood. 2003;101(7):2496-2506.

2. Dispenzieri A. POEMS syndrome. Blood Rev. 2007;21(6):285-299. 
3. Ng AWY, Yuen KK, Tung SY, O SK. Peripheral Neuropathy: Clinical Diagnosis of POEMS Syndrome and Treatment with Radiotherapy. Journal Hong Kong College of Radiologists 2007; 10: 66-69

4. Ozden A, Suleyman Z, Seven G, Arat M, Akin A, Sener O, Kuzu I, et al. POEMS syndrome: a case report. Turk J Gastroenterol. 2008;19(4):276-280.

5. Lesprit P, Godeau B, Authier FJ, Soubrier M, Zuber M, Larroche C, Viard JP, et al. Pulmonary hypertension in POEMS syndrome: a new feature mediated by cytokines. Am J Respir Crit Care Med. 1998;157(3 Pt 1):907-911.

6. Dham A, Peterson BA. Castleman disease. Curr Opin Hematol. 2007;14(4):354-359.

7. Yamasaki S, Iino T, Nakamura M, Henzan H, Ohshima K, Kikuchi M, Otsuka T, et al. Detection of human her- pesvirus- 8 in peripheral blood mononuclear cells from adult Japanese patients with multicentric Castleman's disease. Br J Haematol. 2003;120(3):471-477.

8. Dams K, Meersseman W, Verbeken E, Knockaert DC. A 59-year-old man with shock, polycythemia, and an underlying paraproteinemia. Chest. 2007;132(4):1393-1396.

9. Watanabe O, Maruyama I, Arimura K, Kitajima I, Arimura H, Hanatani M, Matsuo K, et al. Overproduction of vascular endothelial growth factor/vascular permeability factor is causative in Crow-Fukase (POEMS) syndrome. Muscle Nerve. 1998;21(11):1390-1397.

10. Guihot A, Couderc LJ, Agbalika F, Galicier L, Bossi P, Rivaud E, Scherrer A, et al. Pulmonary manifestations of multicentric Castleman's disease in HIV infection: a clinical, biological and radiological study. Eur Respir J. 2005;26(1):118-125. 